\title{
Rare disease knowledge enrichment through a data-driven approach
}

\author{
Feichen Shen ${ }^{*}$ D, Yiqing Zhao, Liwei Wang, Majid Rastegar Mojarad, Yanshan Wang, Sijia Liu and Hongfang Liu*
}

\begin{abstract}
Background: Existing resources to assist the diagnosis of rare diseases are usually curated from the literature that can be limited for clinical use. It often takes substantial effort before the suspicion of a rare disease is even raised to utilize those resources. The primary goal of this study was to apply a data-driven approach to enrich existing rare disease resources by mining phenotype-disease associations from electronic medical record (EMR).

Methods: We first applied association rule mining algorithms on EMR to extract significant phenotype-disease associations and enriched existing rare disease resources (Human Phenotype Ontology and Orphanet (HPOOrphanet)). We generated phenotype-disease bipartite graphs for HPO-Orphanet, EMR, and enriched knowledge base HPO-Orphanet + and conducted a case study on Hodgkin lymphoma to compare performance on differential diagnosis among these three graphs.

Results: We used disease-disease similarity generated by the eRAM, an existing rare disease encyclopedia, as a gold standard to compare the three graphs with sensitivity and specificity as $(0.17,0.36,0.46)$ and $(0.52,0.47,0.51)$ for three graphs respectively. We also compared the top 15 diseases generated by the HPO-Orphanet + graph with eRAM and another clinical diagnostic tool, the Phenomizer.

Conclusions: Per our evaluation results, our approach was able to enrich existing rare disease knowledge resources with phenotype-disease associations from EMR and thus support rare disease differential diagnosis.
\end{abstract}

Keywords: Data-driven approach, Rare disease, Knowledge enrichment, Differential diagnosis

\section{Background}

Rare diseases, although individually rare, collectively affect one in ten Americans. Approximately 7000 rare diseases exist, with more being discovered each year [1]. Patients with rare diseases face diagnostic delay: $40 \%$ of rare disease patients are diagnosed incorrectly before reaching a final diagnosis, of which $25 \%$ spend between 5 to 30 years on a chaotic journey through numerous referrals, investigations, and disease evolutions from early symptoms to a confirmatory diagnosis of their disease [2]. Although there are many genetic tests available for delivering precision medicine, how to identify patients who may benefit from those genetic tests is not obvious. Many rare diseases can be misdiagnosed as common diseases due to their rarity. It often takes substantial clinical time and effort before a rare disease is even a suspected diagnosis [3].

\footnotetext{
* Correspondence: shen.feichen@mayo.edu; liu.hongfang@mayo.edu Department of Health Sciences Research, Mayo Clinic, 205 3rd Ave SW, Rochester, MN 55905, USA
}

The diagnosis pathway of rare diseases is highly dependent on the associated clinical phenotypes, i.e., the observable characteristics, at the physical, morphologic, or biochemical level, of an individual [4]. Symptoms could be treated as phenotypes in symptomatic diagnosis. Taking Hodgkin lymphoma as an example, since symptoms of Hodgkin lymphoma are very similar to other diseases or conditions, such as Cytomegalovirus, Sarcoidosis, and Toxoplasmosis [5], it is meaningful to use underlying disease-phenotype associations to accelerate early differential diagnosis and largely shorten the diagnostic odyssey for patients.

Rare disease knowledge resources exist to assist the diagnosis of rare diseases. For example, the Genetic and Rare Diseases (GARD) resource provides curated information for more than 4700 rare diseases, including their symptoms, causes, inheritance, treatments, and prognoses as well as the latest research [6]. Orphanet [7] provides an expert-vetted and up-to-date encyclopedia of 
rare diseases along with their associated genes. The $\mathrm{Hu}$ man Phenotype Ontology (HPO) [8] provides a controlled vocabulary for clinical phenotypes by mining and integrating clinical phenotype knowledge from literature and a variety of rare disease resources.

Some other existing studies investigated the mining of associations between diseases and genes. For example, Zhang et al. combined the Latent Dirichlet Allocation (LDA) [9] with network-based computational approach [10] to discover disease-gene associations from large amount of PubMed literature [11]. Piro et al. developed a classification approach to predict disease-gene associations [12]. By leveraging a network distance measure and a random walk algorithm, Kohler et al. presented a method to prioritize candidate genes for hereditary disorders [13]. However, all of these studies focused solely on extracting information from literature or knowledge bases. It often takes substantial time and effort before the suspicion of a rare disease is even raised to utilize those resources due to its rarity.

There are some related studies utilizing either electronic medical record (EMR) or literature or both to investigate diseases, phenotypes and their associations. For example, $\mathrm{Xu}$ et al. introduced text mining result of disease-phenotype associations by analyzing sentences from MEDLINE [14]. In another study, Garcelon et al. described a text mining based analysis leveraging TF-IDF to discover associations between clinical phenotypes and rare diseases [15]. Their results showed that phenotypes identified in EMR can be a useful source of evidence to provide rare disease specialists with candidate phenotypes. The eRAM is an encyclopedia of rare disease annotations mined from 10 million scientific publications and EMR [16]. Authors of the eRAM implemented a web-based tool to provide clinicians with next-step information of disease-disease associations in addition to disease-phenotype associations. The tool systematically incorporates disease-phenotype associations of rare diseases from both published medical literatures and clinical data. Hassan et al. investigated on extracting associations between rare diseases and phenotypes to enrich existing ontology [17]. The Phenomizer [18] is a clinical diagnostic tool that aims to help clinicians to identify the potential diagnostic candidates. It is built based on the HPO, Orphanet and Online Mendelian Inheritance in Man (OMIM) [19]. Unfortunately, EMR was not incorporated in $[17,18]$.

Here, we used the HPO annotation file named "phenotype_annotation.tab" accessed in July 2017 for association information between HPO terms and rare diseases in Orphanet [20]. These associations, which we referred to as HPO-Orphanet associations, were treated as rare disease knowledge resource in this study. We propose to enrich the HPO-Orphanet through mining association information between clinical phenotypes and diseases using EMR. Such enriched information, named as HPO-Orphanet+, can be used to link similar rare/common diseases and provide differential diagnostic decision aid at the point of care for rare disease diagnosis.

In the following, we first introduce the methods used in our study. We then describe our experimental evaluations. Results are presented next followed by discussion. We conclude our study with potential future work.

\section{Methods \\ Materials}

All clinical notes during the years of 2010 to 2015 from Mayo Clinic EMR were used for the study, including Consultant Notes (CON), Subsequent Visit Notes (SV), Emergency Medicine Notes (EMV), Hospital Admission Notes (ADM), and so on. For each note type, we focused on the diagnosis section of the notes which summarizes problems for each patient. The resulting corpus contains 12.8 million clinical notes corresponding to 729,000 patients. In our previous work, we have developed a phenotype-disease annotation pipeline that utilized the HPO and the Unified Medical Language System (UMLS) [21] to extract phenotypic and disease terms from clinical narratives [22, 23], where disease and phenotype appeared in the same clinical note was considered to be a phenotype-disease association. Specifically, the HPO was used to identify rare diseases and their phenotypic characterization mentioned in clinical narratives, and the UMLS was utilized to detect synonyms for any phenotypic terms. We limited our annotation to sections containing problems and diagnoses where 38,097 patients were found to have at least one diagnosis of a rare disease. Leveraging this pipeline, we extracted 2808 unique phenotypes from notes and 9,292,969 phenotype-disease associations in total, from which 164,792 associations were related to 1449 rare diseases and the rest were generated from 13,821 common diseases.

Semantic MEDLINE Database (SemMedDB), a repository of semantic predications extracted from the titles and abstracts of all PubMed citations [24-27], was used in this study to quantify the explanatory power of enriched associations. We used SemMedDB Version 25 in this study.

We used the eRAM to build a gold standard on disease differential diagnosis and used the gold standard to evaluate performance among three bipartite graphs. In addition, we compared the top 15 differential diagnostic candidates generated by the HPO-Orphanet+, Phenomizer, and eRAM. Specifically, the HPO-Orphanet+ and eRAM ranked diagnostic candidates by the descending order of Jaccard similarity score [28], and the Phenomizer ranked diagnostic candidates by the descending order of Information Content (IC)-based similarity score proposed in [18]. 


\section{System design}

The overall workflow of our study is shown in Fig. 1. After obtaining raw phenotype-disease pairs from EMR using the previously developed annotation pipeline, we formulated the task of mining association information between clinical phenotypes and diseases as an association rule mining task [29]. Specifically, patients are considered as transactions and their phenotypes and diseases are considered as items. The phenotype-disease association discovery can be defined as one item rule \{Phenotype $\rightarrow$ \{Disease $\}$. We leveraged the support and confidence metrics (Eqs. 1 and 2) to measure the importance of discovered phenotype-disease relationships [30].

Given phenotype $\mathrm{P}$ and disease $\mathrm{D}$, support is calculated as:

$$
\begin{aligned}
& \operatorname{support}(P)=\frac{\text { \#of unique patients having Phenotype } P}{\text { \#of unique patients }} \\
& \operatorname{support}(D)=\frac{\text { \#of unique patients having Disease } D}{\text { \#of unique patients }} \\
& \operatorname{support}(P D)=\frac{\text { \#of unique patients having Phenotype } P \& \text { Disease D }}{\text { \#of unique patients }}
\end{aligned}
$$

confidence is calculated as:

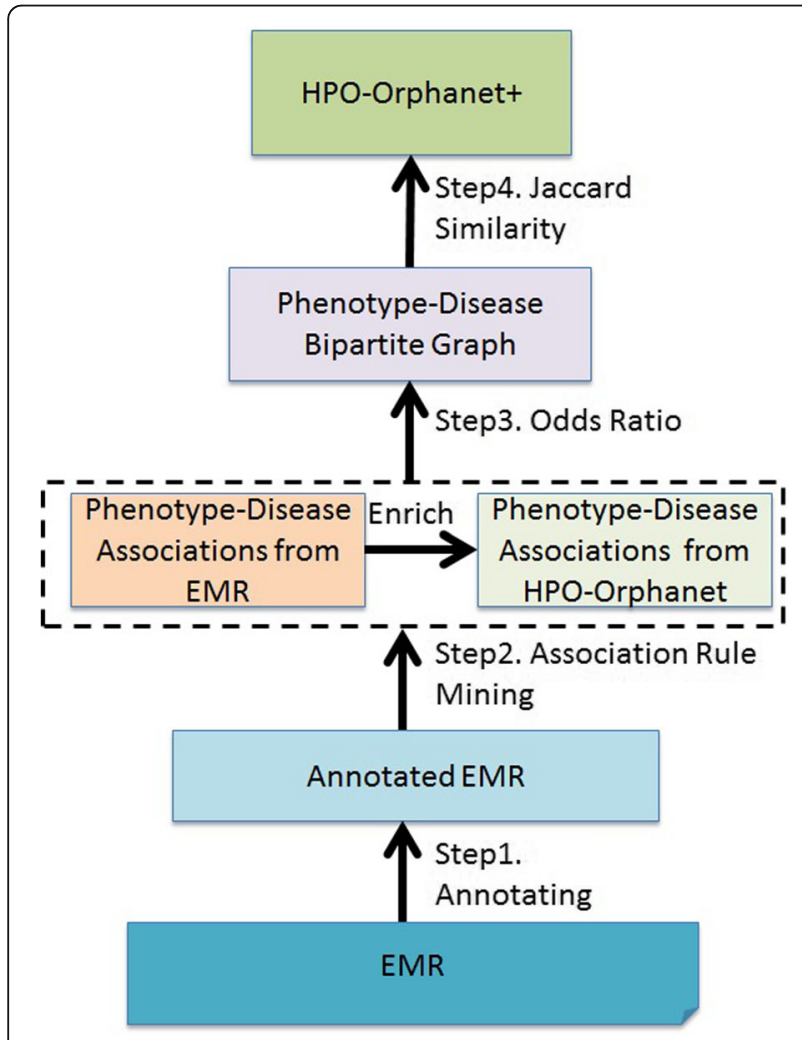

Fig. 1 System workflow

$$
\operatorname{confidence}(P \Rightarrow D)=\frac{\text { support }(\mathrm{PD})}{\text { support }(\mathrm{P})}
$$

In addition, we filtered out less important phenotypedisease associations. In our previous study [31], we have demonstrated the use of odds ratio [32, 33] to detect significant phenotype-disease associations from a huge number of patient data, therefore, in this study, the odds ratio was applied on any disease-phenotype associations to find significant phenotypes for diseases. For any pair of disease $\mathrm{D}$ and phenotype $\mathrm{P}$, the odds ratio $\mathrm{OR}(\mathrm{D}, \mathrm{P})$ is defined as:

$$
\begin{aligned}
& \text { (\#associations contain both } D \text { and } P \text { ) * } \\
& \text { OR }(D, P)=\frac{(\# \text { associations contain neither } D \text { nor } P)}{(\# \text { associations contain } D \text { and phenotypes except } P)} \text { * } \\
& \text { (\#associations contain } P \text { and diseases except D) }
\end{aligned}
$$

In this study, we used a bipartite graph to represent associations between diseases and phenotypes. Given two disjoint and independent sets $\mathrm{U}$ and $\mathrm{V}$, let $\mathrm{U}$ denote disease sets and $\mathrm{V}$ denote phenotype sets, the bipartite graph $G$ is defined as a graph such that each edge connects a vertex in $\mathrm{U}$ to one in $\mathrm{V}$ [34].

Given a collection of phenotype-disease associations $\mathrm{C}$, we implemented a heuristics for generating differential diagnostic candidates. For a disease D, we first selected those phenotypes with the corresponding lower bound of odds ratio values larger than one [35]. A phenotype-disease bipartite graph was then generated. Jaccard similarity was commonly adopted on detecting disease similarity [28], hence in this study, we applied Jaccard similarity on disease level, aiming to measure the similarity among diseases based on significant phenotypic features selected by odds ratio. Given two diseases $D_{i}$ and $D_{j}$, denoting phenotype sets for $D_{i}$ and $D_{j}$ as $\left\{P_{i}\right\}$ and $\left\{P_{j}\right\}$ respectively, the Jaccard similarity J $\left(D_{i}, D_{j}\right)$ is defined as:

$$
J\left(D_{i}, D_{j}\right)=\frac{\left|\left\{P_{i}\right\} \cap\left\{P_{j}\right\}\right|}{\left|\left\{P_{i}\right\} \cup\left\{P_{j}\right\}\right|}
$$

The derived HPO-Orphanet+ graph stored diseases as nodes and weighted edges as Jaccard similarity scores between diseases.

\section{Results}

Evaluation approach

Evaluation of our system comprised of three major components: 1) Enrichment of phenotype-disease associations, 2) Bipartite graph comparison, and 3) Performance on rare disease differential diagnosis. 


\section{Enrichment of phenotype-disease associations}

We used phenotype-disease associations reported in the SemMedDB to quantify the explanatory power of enriched associations mined from EMR. We set average support and average confidence as minimum thresholds to pick top associations. We leveraged the increment of explanatory power (IEP) [36] to quantify the enrichment on HPO-Orphanet.

\section{Increment of explanatory power (IEP)}

We used explanatory power (EP) as defined in the study [36] to represent the associations explained by HPOOrphanet:

$$
E P=\# \text { of associations explained by knowledge base }
$$

We then quantified the knowledge increment by finding the increment of explanatory power (IEP) [36] for the enriched knowledge base generated by our approach compared to the initial HPO-Orphanet annotations:

$$
I E P=\frac{U K_{i}-U K_{n}}{U K_{i}}
$$

where $\mathrm{UK}_{\mathrm{i}}$ is the number of unexplained associations from the initial HPO-Orphanet knowledge and $\mathrm{UK}_{\mathrm{n}}$ is the number of unexplained associations in the enriched knowledge resource HPO-Orphanet+.

\section{Bipartite graph comparison}

We limited our associations to diseases appearing in both EMR and HPO-Orphanet. We compared graph features for three bipartite graphs based on associations mined from EMR alone, HPO-Orphanet alone, and the combination of the prior two, HPO-Orphanet+. Graph characterization measurements we used were defined as follows:

\section{Density}

For undirected simple graphs, the graph density was defined as [37].

$$
\operatorname{Density}(G)=\frac{2|E|}{|V|(|V|-1)}
$$

where $E$ is the number of edges in the graph and $V$ is the number of vertices in the graph.

\section{Average degree}

The average degree of a vertex of a graph is the average number of edges connected to the vertex [38], and is defined as follows:

$$
\bar{\Delta}(G)=\frac{\sum \operatorname{deg}(v)}{|V|}=\frac{2|E|}{|V|}
$$

where $\mathrm{E}$ is the number of edges in the graph and $\mathrm{V}$ is the number of vertices in the graph.

\section{Performance on rare disease differential diagnosis}

We compared performance for generating differential diagnostic candidates among HPO-Orphanet graph, EMR graph, and HPO-Orphanet+ graph.

To prepare the experiment, for any disease to be tested, we used the three aforementioned graphs to rank suggested diseases with descending order of Jaccard similarity score. We combined two disease-phenotype association files namely "eRAM Integrated Phenotype.txt" and "eRAM Integrated Symptom.txt" provided by the eRAM. Since those two files were manually annotated by domain experts, in this study, we considered the associations provided by the merged files as correct ones to prepare the gold standard. Based on 5356 curated diseases and their associated phenotypes/symptoms obtained from the files, we calculated Jaccard similarity score between each pair of diseases using Eq. 4. Such disease-disease similarity was used as a gold standard on differential diagnosis. In this evaluation, we first validated diagnostic candidates generated by the three bipartite graphs using the gold standard. We then compared the top 15 differential diagnostic candidates generated by the HPO-Orphanet+ graph, Phenomizer, and eRAM.

Table 1 shows the confusion matrix for performance evaluation. For any given tested disease, sensitivity and specificity were defined as shown in Eqs. 9 and 10.

$$
\begin{aligned}
& \text { Sensitivity }=\frac{T P}{T P+F N} \\
& \text { Specificity }=\frac{T N}{T N+F P}
\end{aligned}
$$

\section{Evaluation results}

Metrics comparison and optimal threshold selection

Since HPO-Orphanet is a rare disease dominant knowledge resource, we focused on the enrichment of

Table 1 Confusion matrix for performance evaluation

\begin{tabular}{lll}
\hline & $\begin{array}{l}\text { Differential diagnosis candidates in the } \\
\text { eRAM gold standard }\end{array}$ & $\begin{array}{l}\text { Differential diagnosis candidates not in the } \\
\text { eRAM gold standard }\end{array}$ \\
\hline $\begin{array}{l}\text { Differential diagnosis candidates generated } \\
\text { by each graph }\end{array}$ & True Positive (TP) & False Positive (FP) \\
$\begin{array}{l}\text { Differential diagnosis candidates not generated } \\
\text { by each graph }\end{array}$ & False Negative (FN) & True Negative (TN) \\
\hline
\end{tabular}


HPO-Orphanet with associations between phenotypes and rare diseases mined from EMR. To select the optimal associations, we set average support score 5E-06 as the threshold to first select 31,211 frequent itemsets and we then set average confidence score 0.05 as the minimum confidence to finalize 13,742 associations (see Additional file 1). To further validate the selection of thresholds, as shown in Fig. 2, we found that both support and confidence value didn't have much fluctuation after dropping below their average values (the threshold point is marked on the curve).

We also characterized the associations using a heatmap as shown in Fig. 3. Specifically, $\mathrm{x}$-axis indicates support value ranges from $1.24 \mathrm{E}-06$ to $1.37 \mathrm{E}-05$ and $\mathrm{y}$-axis indicates confidence value ranges from 0.0005 to 1 . From Fig. 3, we observed that the number of rules get decreased with the increment of both support and confidence.

\section{Enrichment of phenotype-disease associations}

As shown in Table 2, 379 rare diseases, 324 phenotypes, and 1775 associations were found from EMR but not appeared in HPO-Orphanet.

10,132 and 4742 pairs of associations can be found from 124,989 associations in literature for EMR and HPO-Orphanet respectively. According to Eq. 6, IEP for enrichment of phenotype-disease associations is $4.48 \%$, quantifying the enrichment after EMR mining.

$$
\begin{aligned}
I E P & =\frac{U K_{i}-U K_{n}}{U K_{i}} * 100 \\
& =\frac{(124,989-4,742)-(124,989-10,132)}{(124,989-4,742)} * 100 \\
& =4.48 \%
\end{aligned}
$$

\section{Enrichment of disease differential diagnosis}

Bipartite graph analysis We constructed three bipartite graphs for the HPO-Orphanet, EMR, and HPO-Orphanet+ based on 97 shared diseases. As shown in Table 3, although EMR provided fewer phenotypes than HPOOrphanet, associations between disease and phenotypes were richer, resulting in an enriched bipartite graph after combination. We also observed that EMR graph has a higher density than HPO-Orphanet graph, indicating that some phenotype-disease pairs held too many associations and imbalanced the entire graph density. The density for the HPO-Orphanet+ graph was the lowest among all graphs. The increment of average degree for combined graph indicated that novel phenotype-disease associations were mined from EMR to enrich HPOOrphanet. According to Eqs. 7 and 8, for a given graph G, Density $(\mathrm{G})$ is calculated by $\bar{\Delta}(\mathrm{G}) /(|\mathrm{v}|-1)$. Although the HPO-Orphanet+ held the highest average degree, since vertices got enriched, resulting a relative lower density for the HPO-Orphanet+.

We also listed top 15 diseases with highest degrees for each bipartite graph as shown in Table 4. After combining the two datasets, we found that some diseases with highest degree mined from EMR graph were still dominant in the HPO-Orphanet+ graph, such as multiple myeloma, hodgkin lymphoma, giant cell arteritis, and follicular lymphoma. But some dominant diseases in HPO-Orphanet graph were not ranked high in the HPO-Orphanet+ graph, such as $22 q 11.2$ deletion syndrome, granulomatosis with polyangiitis, and marfan syndrome. In addition, we observed that neurofibromatosis type1 is the one that didn't appear in the top list for either EMR or HPO-Orphanet, denoting that the combination of EMR and HPO-Orphanet enriched the phenotypic sets for neurofibromatosis type1 and thus increased its connectivity.

\section{Rare disease differential diagnostic suggestions - Use case study}

We carried a use case study on Hodgkin lymphoma to compare the performance for three different bipartite
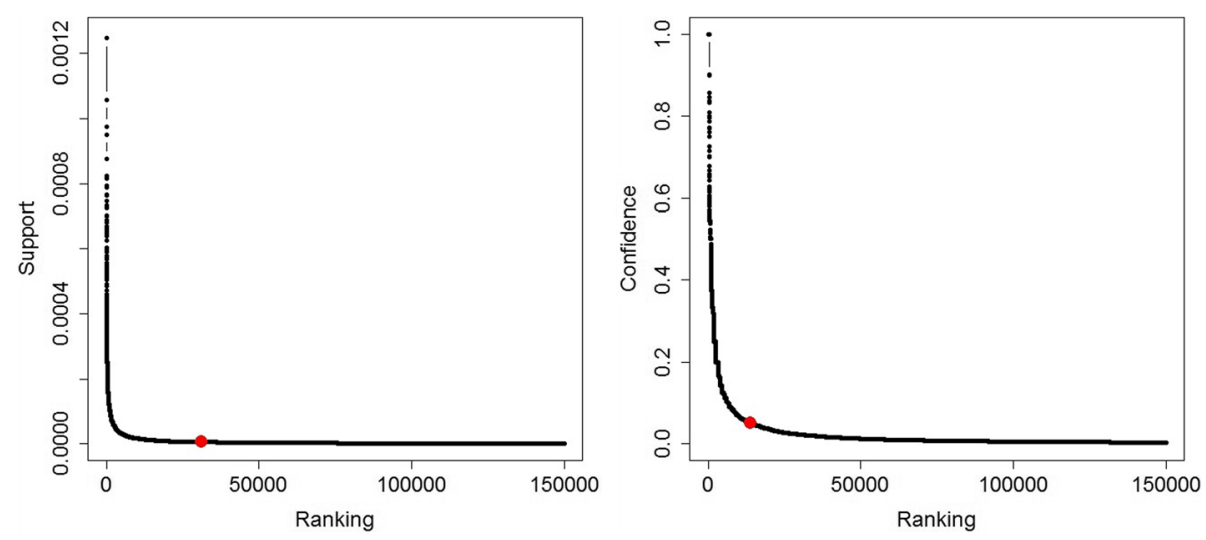

Fig. 2 Plotted curve between association ranking and two metrics 


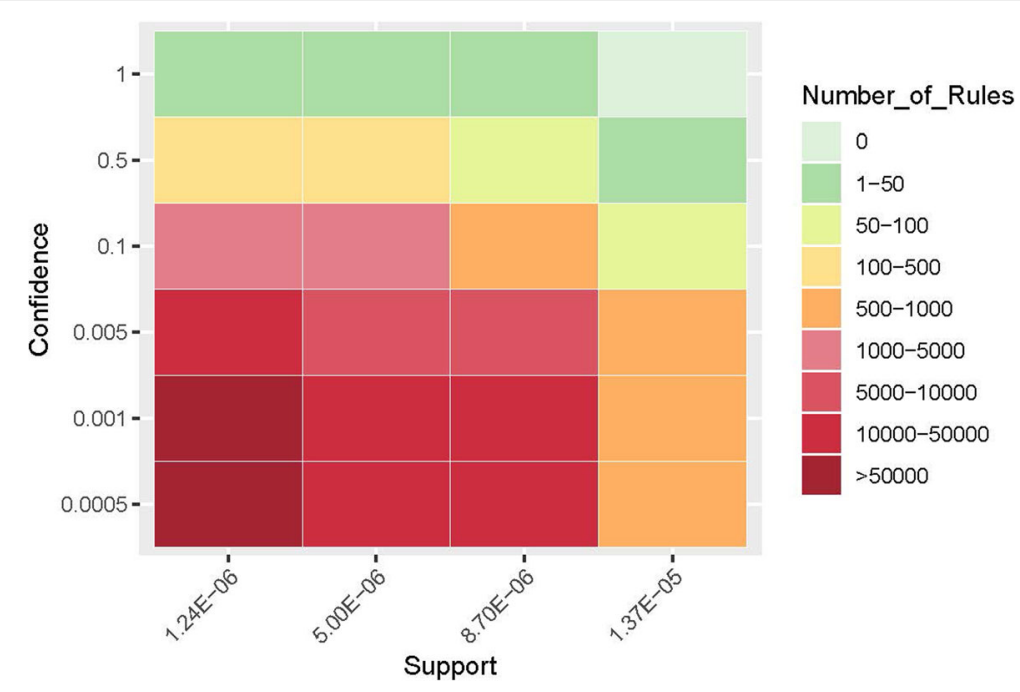

Fig. 3 Characterization of associations

graphs. The number of unique differential diagnostic suggestions for Hodgkin lymphoma generated by HPO-Orphanet, EMR, and HPO-Orphanet+ is 2663, 10,064 , and 11,439 respectively.

Hodgkin lymphoma is a type of lymphoma that results from white blood cells called lymphocytes. Common symptoms related to Hodgkin lymphoma are painless swelling of lymph nodes in neck, armpits or groin, persistent fatigue, fever and chills, night sweats, rapid weight loss, itching, increased sensitivity to the effects of alcohol [39]. Sensitivity and specificity for generating differential diagnostic suggestions for Hodgkin lymphoma with different graphs is shown in Fig. 4. The HPO-Orphanet+

Table 2 Statistics between EMR and HPO-Orphanet on the number of rare diseases, phenotypes, and phenotype-disease associations

\begin{tabular}{ll}
\hline Number of Unique Rare Diseases & Count \\
HPO-Orphanet & 2664 \\
EMR & 476 \\
HPO-Orphanet and EMR & 97 \\
In EMR but not in HPO-Orphanet & 379 \\
Number of Unique Phenotypes & Count \\
HPO-Orphanet & 4577 \\
EMR & 1337 \\
HPO-Orphanet and EMR & 1013 \\
In EMR but not in HPO-Orphanet & 324 \\
Number of Unique Associations & Count \\
HPO-Orphanet & 7529 \\
EMR & 1973 \\
HPO-Orphanet and EMR & 198 \\
In EMR but not in HPO-Orphanet & 1775 \\
\hline
\end{tabular}

graph shows the highest sensitivity for detecting the right similar diseases according to the eRAM gold standard, while using the HPO-Orphanet graph yields the lowest sensitivity. In addition, specificity does not show significant differences among three graphs, indicating that all of them have similar performance on rejecting non-relevant diseases for Hodgkin lymphoma. In general, we observed that the HPO-Orphanet+ graph enriched the existing rare disease knowledge resources and thus be able to provide better diagnostic suggestions. A web-based tool was implemented to visualize diagnostic suggestions and Fig. 5 shows an example of this differential diagnostic decision aid interface by considering Hodgkin lymphoma as a center node.

Table 5 shows top 15 differential diagnostic candidates for Hodgkin lymphoma between the HPO-Orphanet+ graph and two existing diagnostic tools (Phenomizer and eRAM). The HPO-Orphanet+ graph identified 46.7\% (7 out of 15) common diseases and 53.3\% (8 out of 15) rare diseases. Specifically, chronic obstructive airway disease, diabetes mellitus, atrial fibrillation, glaucoma, coronary heart disease, degenerative polyarthritis, and chronic kidney insufficiency are common diseases that share the most similar phenotypes with Hodgkin lymphoma, which are considered to be potential candidates for misdiagnosis of Hodgkin lymphoma. In addition, based on literature and online material review, we found that 10 out of 15 diagnostic candidates were proved to be strongly associated with Hodgkin lymphoma based on similar comorbidities or complications [5, 40-48]. For example, lung adenocarcinoma has similar characterizations with Hodgkin lymphoma [46], and glomerulonephritis is a well-recognized complication of Hodgkin disease [49]. Few evidences were detected for dilated cardiomyopathy, abdominal aortic aneurysm, degenerative polyarthritis, 
Table 3 Graph characterization for bipartite graphs generated from the HPO-Orphanet, EMR, and HPO-Orphanet+ (based on 97 shared diseases)

\begin{tabular}{llll}
\hline & HPO-Orphanet Graph & EMR Graph & HPO-Orphanet+ Graph \\
\hline \# of Disease Nodes & 97 & 97 & 97 \\
\# of Phenotype Nodes & 722 & 670 & 1194 \\
\# of Edges & 1973 & 2071 & 3914 \\
Density & 0.006 & 0.007 & 0.005 \\
Average Degree & 4.818 & 5.4 & 6.064 \\
\hline
\end{tabular}

atrial fibrillation, and glaucoma from online materials and scientific literature, indicating that the associations mined from patients' data provided new evidences for differential diagnosis of Hodgkin lymphoma.

While differential diagnostic candidates provided by the Phenomizer are all rare disease. Similarly, the eRAM generates $93.3 \%$ (14 out of 15) rare diseases but only $6.7 \%$ ( 1 out of 15 ) common diseases.

Since many rare diseases are commonly misdiagnosed as common diseases, it is essential to link common and rare diseases at the early time of diagnosis to assist in diagnostic decision support. Compared to the Phenomizer and eRAM, the HPO-Orphanet+ graph is more capable of detecting such associations.

\section{Discussion}

Our system can benefit the clinical practice by continuously mining knowledge from EMR to make a enriched rare disease knowledge resource incorporating information from both knowledge and data-driven insights, which is currently lacking in other systems [50-52]. However, false positive phenotype-disease relationships contributed by comorbidities are hard to detect. The odds ratio can address this issue to some extent. We will extend our current singleton frequent item set association rule mining to include two items and three items (e.g., bigram and trigram) to better support elimination of false positives. In addition, according to some existing and our previous studies [53-56], we also set thresholds as the average of metrics to select optimal associations. In the future, we will make an optimal threshold selection scheme combining both average value and elbow criterion [57] in association rule mining. Moreover, for those novel disease-phenotype associations mined from data and cannot be validated by biomedical literature, online database or knowledge base, we will recruit domain experts to provide a manual evaluation and curate the enriched knowledge base in the future work. More evaluation metrics (e.g., precision, recall, and F-measure) will be applied based on experts' judgements.

In this study, we extracted the co-occurrence information between a phenotype and a disease from diagnosis section contained in clinical notes. Specifically, we first split the entire notes into sentences and then applied the aforementioned annotation pipeline on each sentence. In addition, problems in those documents are generally

Table 4 Top 15 diseases with the highest degree in bipartite graphs generated from the HPO-Orphanet, EMR, and HPO-Orphanet+

\begin{tabular}{lll}
\hline HPO-Orphanet Graph & EMR Graph & HPO-Orphanet+ Graph \\
\hline 22q11.2 deletion syndrome & multiple myeloma & multiple myeloma \\
melas & hodgkin lymphoma & hodgkin lymphoma \\
granulomatosis with polyangiitis & follicular lymphoma & giant cell arteritis \\
marfan syndrome & giant cell arteritis & follicular lymphoma \\
neurofibromatosis type 1 & primary sclerosing cholangitis & primary sclerosing cholangitis \\
trisomy 18 & myasthenia gravis & 22q11.2 deletion syndrome \\
eosinophilic granulomatosis with polyangiitis & granulomatosis with polyangiitis & granulomatosis with polyangiitis \\
giant cell arteritis & pulmonary arterial hypertension & melas \\
acromegaly & liposarcoma & myasthenia gravis \\
primary sclerosing cholangitis & eosinophilic esophagitis & rheumatic fever \\
systemic sclerosis & rheumatic fever & marfan syndrome \\
dermatomyositis & klatskin tumor & dermatomyositis \\
osteogenesis imperfecta & tetralogy of fallot & pulmonary arterial hypertension \\
addison disease & cystic fibrosis & craniopharyngioma \\
cushing syndrome & craniopharyngioma & neurofibromatosis type1 \\
\hline
\end{tabular}




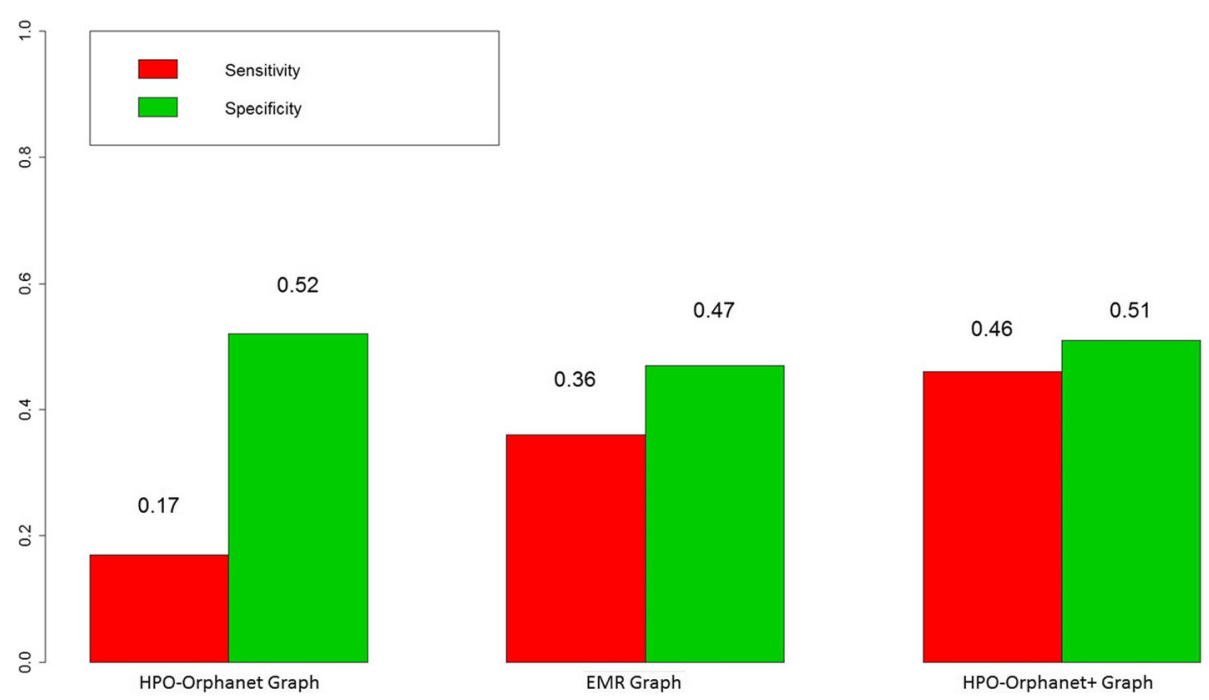

Fig. 4 Comparison on differential diagnostic suggestion performance for Hodgkin Lymphoma

itemized entries as either phrases (e.g., Allergic rhinitis/ vasomotor rhinitis) or short sentences (e.g, Her asthma appeared to be very mild), therefore, we didn't use window size to limit the distance between phenotype and disease. In the future, to generalize the association mining on larger size of documents, we will seek to investigate the selection of appropriate window size for a better performance [58]. Moreover, some network analysis approaches [59] with knowledge network discovery algorithms $[60,61]$ will be incorporated with association rule mining to reveal hidden relations among diseases.
We used the SemMedDB to measure the IEP of knowledge enrichment. However, some evidences indicated that the SemMedDB is not so accurate due to the limitation of the extraction algorithms used. For example, the SemRep (the generator for SemMedDB) yielded about $75 \%$ precision on information extraction [62]. In the future, we will incorporate more disease and phenotype knowledge bases with human annotated associations to measure the knowledge enrichment.

We compared the HPO-Orphanet+ with both the Phenomizer and eRAM in this study on differential diagnostic

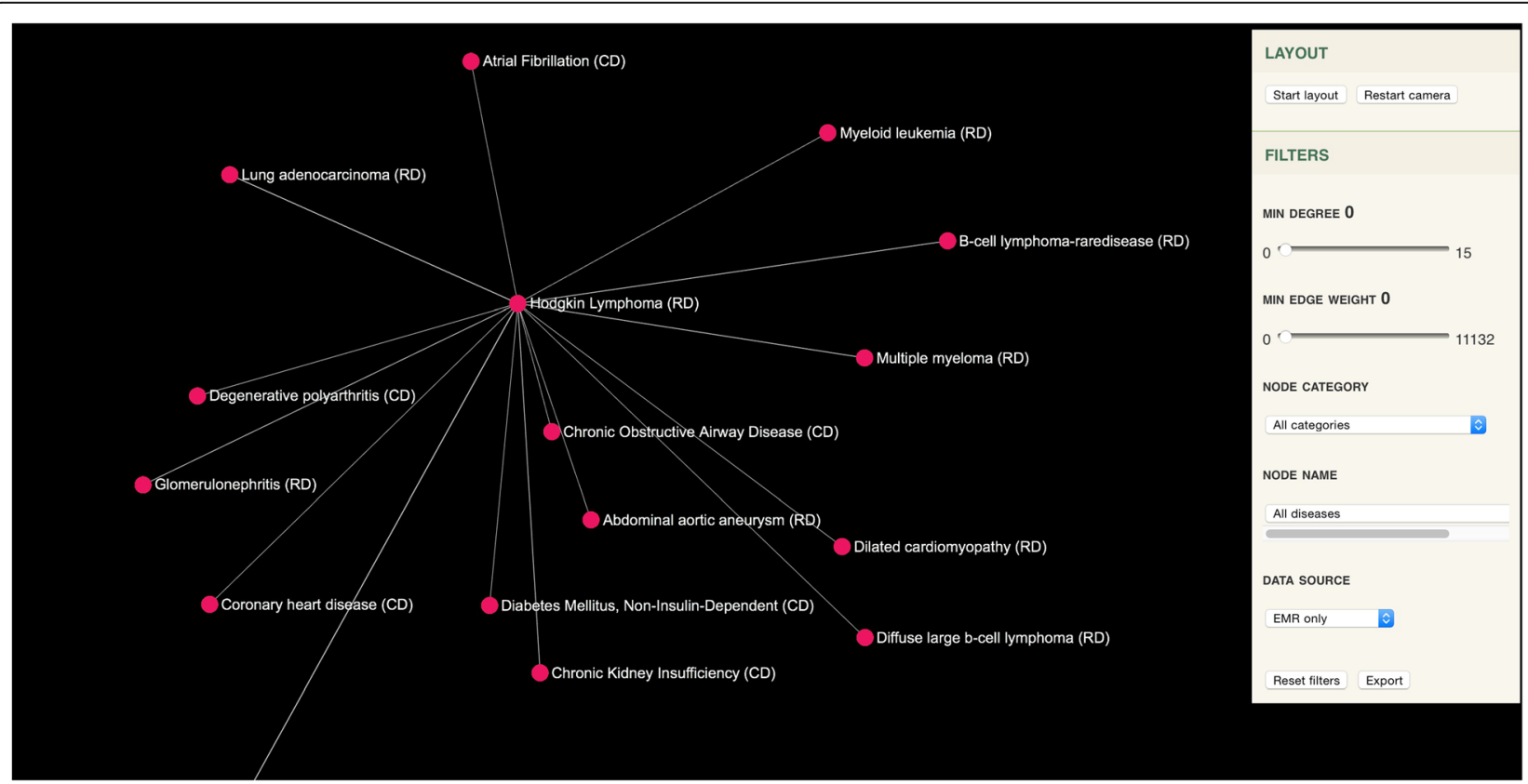

Fig. 5 Interactive web-based tool for differential diagnostic suggestion (CD stands for Common Disease, and RD stands for Rare Disease) 
Table 5 Top 15 differential diagnostic candidates for the HPO-Orphanet+ graph, Phenomizer, and eRAM on Hodgkin lymphoma. Scores in column 1 and 3 indicate Jaccard similarity and scores in column 2 indicate the IC-based score calculated by the Phenomizer (CD stands for common disease, and RD stands for rare disease)

\begin{tabular}{|c|c|c|}
\hline HPO-Orphanet+ Graph & Phenomizer & eRAM \\
\hline B-cell lymphoma (RD): 0.626 & Classic hodgkin lymphoma (RD): 3.986 & $\begin{array}{l}\text { Nodular lymphocyte predominant hodgkin } \\
\text { lymphoma (RD): } 0.458\end{array}$ \\
\hline Diffuse large b-cell lymphoma (RD): 0.62 & Behcet syndrome (RD): 3.189 & Schnitzler syndrome (RD): 0.273 \\
\hline Chronic Obstructive Airway Disease (CD): 0.595 & Aggressive systemic mastocytosis (RD): 3.176 & Mantle cell lymphoma (RD): 0.25 \\
\hline Dilated cardiomyopathy (RD): 0.594 & Alveolar echinococcosis (RD): 3.085 & Pulmonary blastoma (RD): 0.25 \\
\hline Abdominal aortic aneurysm (RD): 0.592 & Systemic lupus erythematosus (RD): 2.997 & Aggressive systemic mastocytosis (RD): 0.22 \\
\hline Glomerulonephritis (RD): 0.591 & Legionellosis (RD): 2.878 & Anemia, autoimmune hemolytic (RD): 0.219 \\
\hline $\begin{array}{l}\text { Diabetes Mellitus, Non-Insulin-Dependent } \\
\text { (CD): } 0.588\end{array}$ & Takayasu arteritis (RD): 2.731 & Hughes syndrome (RD): 0.219 \\
\hline Multiple myeloma (RD): 0.588 & Cystic echinococcosis (RD): 2.648 & Follicular lymphoma (RD): 0.214 \\
\hline Atrial Fibrillation (CD): 0.585 & Eosinophilic granuloma (RD): 2.647 & Thymic carcinoma (RD): 0.214 \\
\hline Glaucoma (CD): 0.58 & Whipple disease (RD): 2.638 & Mast cell sarcoma (RD): 0.2 \\
\hline Myeloid leukemia (RD): 0.58 & Familial thrombocytosis (RD): 2.632 & American trypanosomiasis (CD): 0.2 \\
\hline Coronary heart disease (CD): 0.58 & Systemic mastocytosis (RD): 2.622 & Alpha-heavy chain disease (RD): 0.194 \\
\hline Degenerative polyarthritis (CD): 0.573 & Emberger syndrome (RD): 2.549 & Klatskin tumor (RD): 0.192 \\
\hline Lung adenocarcinoma (RD): 0.572 & Hypocomplementemic urticarial vasculitis (RD): 2.548 & Legionellosis (RD): 0.189 \\
\hline Chronic Kidney Insufficiency (CD): 0.571 & Babesiosis (RD): 2.499 & Babesiasis (RD): 0.182 \\
\hline
\end{tabular}

suggestions. Results showed that the HPO-Orphanet+ is capable of providing a diagnostic graph mixed with both rare and common diseases, which has potential usage in rare disease differential diagnosis, especially for those rare diseases sharing similar symptoms with common diseases. In the future, we will upgrade the HPO-Orphanet+ by mining disease-gene information from literature [11, 63]. In addition, one recent research proposed a novel idea by introducing the concept of "property" as a third layer in addition to traditional two-layer disease-phenotype relationship [64]. This study was able to calculate the probability of getting specific diseases from a multisymptom Naïve Bayes algorithm. The third layer of "property" or multisymptoms is an interesting concept that may be involved in our future work.

\section{Conclusions}

In this study, we proposed a data-driven approach to mine phenotype-disease associations buried in EMR so as to enrich current rare disease knowledge with newly extracted associations as well as differential diagnostic suggestions.

\section{Additional file}

Additional file 1: Top Associations. This file includes 13,742 top phenotype-disease associations selected by support and confidence. (XLSX $218 \mathrm{~kb})$

\section{Abbreviations}

CD: Common Disease; EMR: Electronic Medical Record; EP: Explanatory Power; FN: False Negative; FP: False Positive; GARD: Genetic And Rare
Diseases; HPO: Human Phenotype Ontology; HPO-Orphanet: Original HPOOrphanet knowledge resource; HPO-Orphanet+: Enriched HPO-Orphanet knowledge resource by EMR mining; IEP: Increment Of Explanatory Power; NLP: Natural Language Processing; OMIM: Online Mendelian Inheritance In Man; RD: Rare Disease; SemMedDB: Semantic Medline Database; TN: True Negative; TP: True Positive; UMLS: Unified Medical Language System

\section{Acknowledgements}

This work has been supported by the National Institute of Health $(\mathrm{NIH})$ grant U01TR0062-1 and TR02019, and the Rare Kidney Stone Consortium (U54DK083908). The Rare Kidney Stone Consortium (U54DK083908) is part of Rare Diseases Clinical Research Network (RDCRN), an initiative of the Office of Rare Diseases Research (ORDR), NCATS. This consortium is funded through collaboration between NCATS, and the National Institute of Diabetes and Digestive and Kidney Diseases. The funders had no role in study design, data collection and analysis, decision to publish, or preparation of the manuscript. Its contents are solely the responsibility of the authors and do not necessarily represent the official views of the funders.

\section{Funding}

This work has been supported by the National Institute of Health $(\mathrm{NIH})$ grant U01TR0062-1 and TR02019, and the Rare Kidney Stone Consortium (U54DK083908).

\section{Availability of data and materials}

Top phenotype-disease associations selected by support are provided as "Additional file 1". Other datasets used and/or analyzed during the current study are available from the corresponding author on reasonable request.

\section{Authors' contributions}

All co-authors are justifiably credited with authorship, according to the authorship criteria. Final approval is given by each co-author. In detail: FS led the research, designed and implemented the system, performed all data analysis and interpretation of results. YZ participated in the conception, design and implementation of the study. LW, MM, YW, and SL made substantial contributions to data acquisition. $\mathrm{HL}$ conceptualized the research made substantial contributions to system design and data interpretation. FS drafted the manuscript. YZ made substantial contributions to the manuscript. LW, MM, YW, and SL critically revised the manuscript. HL confirmed approach and provided essential editorial support on the manuscript. 


\section{Ethics approval and consent to participate}

This study used existing records to conduct a retrospective study. The study and a waiver of informed consent were approved by Mayo Clinic Institutional Review Board in accordance with 45 CFR 46.116 (Approval \#17-003030).

\section{Consent for publication}

The author(s) declare(s) that the manuscript does not contain any individual person's data. So this paper requires no consent to publish.

\section{Competing interests}

The authors declare that they have no competing interests.

\section{Publisher's Note}

Springer Nature remains neutral with regard to jurisdictional claims in published maps and institutional affiliations.

\section{Received: 13 August 2018 Accepted: 1 February 2019 Published online: 14 February 2019}

\section{References}

1. Boat TF, Field MJ. Rare diseases and orphan products: accelerating research and development. Washington, D.C.: National Academies Press; 2011.

2. Survey of the delay in diagnosis for 8 rare diseases in Europe. Available at: https://wwweurordisorg/sites/default/files/publicationsFact_Sheet_ Eurordiscare2pdf.

3. Rare Diseases Difficult to Diagnose, Cures Hard to Come By. Available at: https://news.aamc.org/research/article/rare-diseases-difficult-diagnose-cureshard-come/.

4. Phenotype Definition. Available at: http://medical-dictionary thefreedictionary.com/phenotype.

5. Hodgkin Lymphoma Differential Diagnosis. Available at: https://emedicine medscape.com/article/201886-differential.

6. Genetic and Rare Diseases Information Center. Available at: https:// rarediseases.info.nih.gov/.

7. Maiella S, Rath A, Angin C, Mousson F, Kremp O. Orphanet and its consortium: where to find expert-validated information on rare diseases. Rev Neurol. 2013;169:S3-8.

8. Robinson PN, Köhler S, Bauer S, Seelow D, Horn D, Mundlos S. The human phenotype ontology: a tool for annotating and analyzing human hereditary disease. Am J Hum Genet. 2008;83(5):610-5.

9. Blei DM, Ng AY, Jordan Ml. Latent dirichlet allocation. J Mach Learn Res. 2003;3:993-1022

10. Barabási A-L, Gulbahce N, Loscalzo J. Network medicine: a network-based approach to human disease. Nat Rev Genet. 2011;12(1):56.

11. Zhang Y, Shen F, Mojarad MR, Li D, Liu S, Tao C, Yu Y, Liu H. Systematic identification of latent disease-gene associations from PubMed articles. PLoS One. 2018;13(1):e0191568.

12. Piro RM, Di Cunto F. Computational approaches to disease-gene prediction: rationale, classification and successes. FEBS J. 2012:279(5):678-96.

13. Köhler S, Bauer S, Horn D, Robinson PN. Walking the interactome for prioritization of candidate disease genes. Am J Hum Genet. 2008:82(4):949-58.

14. Xu R, Li L, Wang Q. Towards building a disease-phenotype knowledge base: extracting disease-manifestation relationship from literature. Bioinformatics. 2013:29(17):2186-94

15. Garcelon N, Neuraz A, Salomon R, Bahi-Buisson N, Amiel J, Picard C, Mahlaoui N, Benoit V, Burgun A, Rance B. Next generation phenotyping using narrative reports in a rare disease clinical data warehouse. Orphanet J Rare Dis. 2018;13(1):85

16. Jia J, An Z, Ming Y, Guo Y, Li W, Liang Y, Guo D, Li X, Tai J, Chen G. eRAM: encyclopedia of rare disease annotations for precision medicine. Nucleic Acids Res. 2017;46(D1):D937-43.

17. Hassan M. Knowledge discovery considering domain Litterature and ontologies: application to rare diseases. Université de Lorraine: Doctoral dissertation; 2017.

18. Köhler S, Schulz MH, Krawitz P, Bauer S, Dölken S, Ott CE, Mundlos C, Horn D, Mundlos S, Robinson PN. Clinical diagnostics in human genetics with semantic similarity searches in ontologies. Am J Hum Genet. 2009:85(4):457-64.

19. Hamosh A, Scott AF, Amberger JS, Bocchini CA, McKusick VA. Online Mendelian inheritance in man (OMIM), a knowledgebase of human genes and genetic disorders. Nucleic Acids Res. 2005;33(suppl_1):D514-7.
20. HPO Annotation File. Available at: http://compbio.charite.de/jenkins/job/ hpo.annotations/lastStableBuild/.

21. Bodenreider $\mathrm{O}$. The unified medical language system (UMLS): integrating biomedical terminology. Nucleic Acids Res. 2004:32(suppl_1):D267-70.

22. Shen F, Liu S, Wang Y, Wang L, Afzal N, Liu H. Leveraging collaborative filtering to accelerate rare disease diagnosis. In: AMIA Annual Symposium Proceedings: 2017: American medical informatics association; 2017. p. 1554.

23. Shen F, Liu S, Wang Y, Wen A, Wang L, Liu H. Utilization of electronic medical records and biomedical literature to support the diagnosis of rare diseases using data fusion and collaborative filtering approaches. JMIR Med Inform. 2018:6(4):e11301.

24. Kilicoglu H, Fiszman M, Rodriguez A, Shin D, Ripple A, Rindflesch TC. Semantic MEDLINE: a web application for managing the results of PubMed searches. In: Proceedings of the third international symposium for semantic mining in biomedicine: 2008; 2008. p. 69-76.

25. Kilicoglu H, Rosemblat G, Fiszman M, Rindflesch TC. Constructing a semantic predication gold standard from the biomedical literature. BMC Bioinformatics. 2011;12(1):486.

26. Kilicoglu H, Shin D, Fiszman M, Rosemblat G, Rindflesch TC. SemMedDB: a PubMed-scale repository of biomedical semantic predications. Bioinformatics. 2012;28(23):3158-60.

27. Rindflesch TC, Fiszman M. The interaction of domain knowledge and linguistic structure in natural language processing: interpreting hypernymic propositions in biomedical text. J Biomed Inform. 2003;36(6):462-77.

28. Mathur S, Dinakarpandian D. Finding disease similarity based on implicit semantic similarity. J Biomed Inform. 2012;45(2):363-71.

29. Agrawal R, Srikant R. Fast algorithms for mining association rules. In: Proc 20th int conf very large data bases, VLDB: 1994; 1994. p. 487-99.

30. Rudin W. Real and complex analysis: Tata McGraw-hill education; 2006.

31. Shen F, Wang L, Liu H. Phenotypic analysis of clinical narratives using human phenotype ontology. Stud Health Technol Inform. 2017;245:581-5.

32. Cornfield J. A method of estimating comparative rates from clinical data. Applications to cancer of the lung, breast, and cervix. J Natl Cancer Inst. 1951;11(6):1269-75.

33. Shen F, Wang L, Liu H. Using human phenotype ontology for phenotypic analysis of clinical notes. Stud Health Technol Inform. 2017;245:1285.

34. Asratian AS, Denley TM, Häggkvist R. Bipartite graphs and their applications, vol. 131. Cambridge: Cambridge University Press; 1998.

35. Szumilas M. Explaining odds ratios. J Can Acad Child Adolesc Psychiatry. 2010;19(3):227.

36. Perera S, Henson C, Thirunarayan K, Sheth A, Nair S. Semantics driven approach for knowledge acquisition from emrs. IEEE J Biomed Health Inform. 2014;18(2):515-24.

37. Coleman TF, Moré JJ. Estimation of sparse Jacobian matrices and graph coloring blems. SIAM J Numer Anal. 1983;20(1):187-209.

38. Diestel R. Graph theory 3rd ed. Graduate texts in mathematics; 2005. p. 173.

39. Hodgkin's Lymphoma. Available at: https://www.mayoclinic.org/diseasesconditions/hodgkins-lymphoma/symptoms-causes/syc-20352646.

40. What Is B-Cell Lymphoma. Available at: https://www webmd.com/cancer/ lymphoma/what-is-b-cell-lymphoma - 1.

41. Risk of Diabetes Higher Among Survivors of Hodgkin Lymphoma. Available at: https://connection.asco.org/magazine/exclusive-coverage/risk-diabeteshigher-among-survivors-hodgkin-lymphoma.

42. Huang $C$, Zhao G, Wang L, Zhang H, Wu X, Zhang M, Ma R, Wang L, Liu Y, Liu L. Simultaneous occurrence of Hodgkin's lymphoma and multiple myeloma: a case report and review of the literature. Oncol Lett. 2016;11(6): 4139-43.

43. Lynn W, Marcus R. Glomerulonephritis preceding late relapse of Hodgkin's disease. Postgrad Med J. 1988;64(751):395-7.

44. Schonfeld SJ, Gilbert ES, Dores GM, Lynch CF, Hodgson DC, Hall P, Storm H, Andersen A, Pukkala E, Holowaty E. Acute myeloid leukemia following Hodgkin lymphoma: a population-based study of 35511 patients. J Nat Cancer Inst. 2006;98(3):215-8

45. van Nimwegen FA, Ntentas G, Darby SC, Schaapveld M, Hauptmann M, Lugtenburg PJ, Janus CP, Daniels L, van Leeuwen FE, Cutter DJ. Risk of heart failure in survivors of Hodgkin lymphoma: effects of cardiac exposure to radiation and anthracyclines. Blood. 2017. https://doi.org/10.1182/blood2016-09-740332.

46. Schoenfeld J, Mauch P, Das P, Silver B, Marcus K, Stevenson M, Ng A. Lung malignancies after Hodgkin lymphoma: disease characteristics, detection methods and clinical outcome. Ann Oncol. 2011;23(7):1813-8. 
47. Luciano RL, Brewster UC. Kidney involvement in leukemia and lymphoma. Adv Chronic Kidney Dis. 2014;21(1):27-35.

48. Hospital Medicine-Hodgkins Lymphoma. Available at: https://www. cancertherapyadvisor.com/hospital-medicine/hodgkins-lymphoma/article/ 601575/.

49. Plager J, Stutzman L. Acute nephrotic syndrome as a manifestation of active Hodgkin's disease: report of four cases and review of the literature. Am J Med. 1971;50(1):56-66.

50. Girdea M, Dumitriu S, Fiume M, Bowdin S, Boycott KM, Chénier S, Chitayat D, Faghfoury H, Meyn MS, Ray PN. PhenoTips: patient phenotyping software for clinical and research use. Hum Mutat. 2013;34(8):1057-65.

51. Köhler S, Doelken SC, Mungall CJ, Bauer S, Firth HV, Bailleul-Forestier I, Black GC, Brown DL, Brudno M, Campbell J. The human phenotype ontology project: linking molecular biology and disease through phenotype data. Nucleic Acids Res. 2013:42(D1):D966-74.

52. Winther $\mathrm{O}$, Svenstrup D, Henningsen PP, Kristiásson R, Jørgensen HL. FindZebra - the search engine for difficult medical cases. Orphanet journal of rare diseases. 2014;9(Suppl 1):05.

53. Shen F, Lee Y. Knowledge discovery from biomedical ontologies in cross domains. PLoS One. 2016;11(8):e0160005.

54. Romero C, Romero JR, Luna JM, Ventura S. Mining rare association rules from e-learning data. In: 3rd international conference on educational data mining: 2010; 2010.

55. Hristovski D, Stare J, Peterlin B, Dzeroski S. Supporting discovery in medicine by association rule mining in Medline and UMLS. Stud Health Technol Inform. 2001;2:1344-8.

56. Shen F, Liu H, Sohn S, Larson DW, Lee Y. BmQGen: biomedical query generator for knowledge discovery. In: Bioinformatics and Biomedicine (BIBM), 2015 IEEE International Conference on: 2015: IEEE; 2015. p. 1092-7.

57. Thorndike RL. Who belongs in the family? Psychometrika. 1953;18(4):267-76.

58. Spence DP, Owens KC. Lexical co-occurrence and association strength. J Psycholinguist Res. 1990;19(5):317-30.

59. Belyi E, Giabbanelli PJ, Patel I, Balabhadrapathruni NH, Abdallah AB, Hameed W, Mago VK. Combining association rule mining and network analysis for pharmacosurveillance. J Supercomput. 2016;72(5):2014-34.

60. Zhu Q, Tao C, Shen F, Chute CG. Exploring the pharmacogenomics knowledge base (pharmgkb) for repositioning breast cancer drugs by leveraging web ontology language (OWL) and cheminformatics approaches. In: Biocomputing 2014. Singapore: World Scientific; 2014. p. 172-82.

61. Shen F, Liu H, Sohn S, Larson DW, Lee Y. Predicate oriented pattern analysis for biomedical knowledge discovery. Intell Inf Manag. 2016;8(3):66.

62. Kilicoglu H, Fiszman M, Rosemblat G, Marimpietri S, Rindflesch TC. Arguments of nominals in semantic interpretation of biomedical text. In: Proceedings of the 2010 workshop on biomedical natural language processing: 2010: Association for Computational Linguistics; 2010. p. 46-54.

63. Zhang Y, Li D, Tao C, Shen F, Liu H. An integrative computational approach to identify disease-specific networks from PubMed literature information. In: Bioinformatics and Biomedicine (BIBM), 2013 IEEE International Conference on: 2013: IEEE; 2013. p. 72-5.

64. Jiang Y, Qiu B, Xu C, Li C. The research of clinical decision support system based on three-layer knowledge base model. J Healthc Eng. 2017;2017: 6535286 .

\section{Ready to submit your research? Choose BMC and benefit from:}

- fast, convenient online submission

- thorough peer review by experienced researchers in your field

- rapid publication on acceptance

- support for research data, including large and complex data types

- gold Open Access which fosters wider collaboration and increased citations

- maximum visibility for your research: over $100 \mathrm{M}$ website views per year

At BMC, research is always in progress.

Learn more biomedcentral.com/submissions 